\title{
IMAGE GUIDED NEUROSURGERY: PRESENT STATE AND PERSPECTIVES
}

\author{
GIOVANNI BROGGI *, IVANO DONES *, PAOLO FERROLI *, \\ ANGELO FRANZINI *, MARCELLO MARCHETTI *, CARLO MARRAS *
}

ABSTRACT. - The image guided neurosurgery was made possible by the great developments of modern neuroradiology whose origins could be identify in UK on 1974 when Hounsfield invented the computer tomography (CT Scan) and on 1980 Lauterbur developed the first magnetic resonance (MR) allowing the direct visualization of the brain.

The term neuronavigation is used to describe the set of computer assisted technologies used by neurosurgeons to guide or "navigate" within the brain. The set of hardware for these purposes is referred to as a "neuronavigator". Since the beginning the purpose of this technology was to create a mathematical model describing a proposed coordinate system for the space within the skull. This "fiducial spatial coordinate system" is used as a reference to describe the position of specific structures within this arbitrary defined space with high accuracy. Robust computer technologies made possible the real-time quantitative spatial fusion of images of the patient's brain with the 3D instantaneous position of surgical instruments or probes detected by motion capture technologies guiding the surgeon to selected targets.

The experiences and the results obtained by these new approaches at the Istituto Neurologico Besta are illustrated and the expected developments in the near future are discussed. Milano.

* Dipartimento di Neurochirurgia, Istituto Neurologico Nazionale "Carlo Besta", 


\section{INTRODUZIONE}

Le moderne metodiche neuroradiologiche, come la TC e la RM, e lo sviluppo di software sempre più sofisticati hanno modificato in maniera radicale il trattamento chirurgico delle patologie del sistema nervoso centrale (SNC). Lo sviluppo della chirurgia guidata da immagini ha permesso un'evoluzione delle procedure mini-invasive tale da garantire il raggiungimento di risultati prima impensabili.

La storia della neuronavigazione ha le sue radici nella fine del XIX secolo quando Roentgen nel 1895 mise a punto un sistema di imaging che utilizzava radiazioni ionizzanti (raggi X). Successivi sviluppi si sono avuti nella prima metà del XX secolo; Spiegel e Wycis negli anni '50 iniziarono ad utilizzare immagini radiologiche ortogonali biplanari intraoperatorie nella scelta dell'approccio chirurgico [1]. Gli stessi autori, in riferimento alle osservazioni fatte da Horsley e Clark nel 1908, secondo le quali ogni millimetro cubo del cervello poteva essere registrato e studiato, crearono il primo casco stereotassico utilizzato sull'uomo. Dopo pochi anni Leksell mise a punto il casco stereotassico che porta il suo nome e che trova ancora oggi applicazione in tutte le metodiche stereotassiche. Con la tecnica stereotassica è infatti possibile calcolare la posizione di qualsiasi punto che sia all'interno di un sistema rigido solidale con la testa attraverso la determinazione delle coordinate sui tre piani spaziali: $\mathrm{x}, \mathrm{y}, \mathrm{z}$.

L'uso del fluoroscopio portatile ha reso possibile, a partire dagli anni '70, la visualizzazione di differenti strutture anatomiche garantendo un supporto intraoperatorio nella chirurgia della colonna vertebrale, dei ventricoli cerebrali, nella chirurgia vascolare e nelle procedure percutanee di approccio al ganglio di Gasser nel trattamento della nevralgia trigeminale.

La neuroradiologia moderna nasce nel Regno Unito; nel 1974 Hounsfield progettò la Tomografia Computerizzata (TC) che, per la prima volta, permetteva la visualizzazione diretta del contenuto endocranico un tempo possibile solamente attraverso immagini indirette fornite dalla ventricolografia e dall'angiografia. Queste due ultime procedure, nate intorno agli anni trenta, consentivano di visualizzare la deformazione o lo spostamento (determinato da una patologia ostruttiva o esercitante effetto massa) dei ventricoli o dei vasi cerebrali attraverso la somministrazione di un mezzo di contrasto visibile ai raggi X. Nel 1980 l'inglese Lauterbur, grazie all'attivazione dei tessuti biologici da parte 
delle onde elettromagnetiche, creò la prima Risonanza Magnetica. Lo sviluppo dei moderni sistemi digitali ha permesso un progressivo miglioramento delle immagini TC e RM, non solo in termini qualitativi e morfologici, ma anche dinamici e funzionali permettendo la visualizzazione di distinti apparati (vascolare, liquorale), di un evento fisiologico meccanico, idrodinamico, di poter rendere visibile una funzione cerebrale o descrivere un evento metabolico. Esistono altre metodiche di Medicina Nucleare che prevedono l'uso di radiotraccianti in grado di studiare il metabolismo cerebrale; l'uso dell'FDG o del PAO nelle metodiche PET (Tomografia ad Emissione di Protone) e SPECT (Tomografia Computerizzata ad Emissione di Protone Singolo) sono in grado di documentare aree di aumentato o diminuito metabolismo cerebrale consentendo la localizzazione di aree ischemiche secondarie a eventi ostruttivi vascolari o di vasospasmo (post emorragico o post traumatico) e di aree tumorali.

La Neurochirurgia è una branca chirurgica piuttosto recente con una storia di soli 70 anni. I padri di questa disciplina [2] fondavano i principi del trattamento delle lesioni cerebrali attraverso una profonda conoscenza dell'anatomia dopo secoli di storia di dissezione anatomica iniziata, in epoca rinascimentale, con i magnifici studi compiuti da Leonardo da Vinci. L'esperienza di Dandy e Cushing degli anni '30 permetteva il miglioramento degli approcci chirurgici descritti negli anni '70 e '80 da Ojenamm, Al Mefty e Yasargil che in quel periodo inserirono l'uso sistematico del microscopio operatorio con evidenti vantaggi nell'approccio di strutture non facilmente individuabili in visione macroscopica.

Il neuronavigatore ha radicalmente mutato l'approccio chirurgico dei gliomi [3-4]. Tradizionalmente il tumore veniva esposto attraverso ampie craniotomie che permettevano il corretto orientamento spaziale con la definizione dei rapporti tra la lesione e le strutture contigue; dopo un'ampia apertura durale, il tumore poteva essere circoscritto attraverso la dissezione del parenchima circostante macroscopicamente sano e quindi asportato. Il neuronavigatore, risolvendo ogni problema di orientamento, permette l'esposizione della lesione attraverso piccole craniotomie e la sua asportazione, agendo esclusivamente all'interno del tessuto patologico rispettando il tessuto nervoso circostante. Anche lesioni estese in profondità o verso aree eloquenti possono essere affrontate poiché l'exeresi si interrompe quando il navigatore indica la prossimità di aree funzionalmente importanti. 
RUOLO DELLA NEURONAVIGAZIONE NEL TRATTAMENTO DI LESIONI DEL SNC

Nonostante le nuove conoscenze nate dalle acquisizioni di biologia molecolare, la nascita di nuovi farmaci e le sempre più complesse metodiche di bioingegneria, la neurochirurgia ha un ruolo centrale nel trattamento delle patologie del sistema nervoso centrale e in particolare nella cura dei tumori cerebrali. L'asportazione chirurgica rappresenta la soluzione terapeutica di prima scelta nel trattamento dei tumori cerebrali in quanto è in grado di garantire una migliore prognosi del paziente. Lo scopo della chirurgia consiste nell'asportazione quanto più radicale della lesione mantenendo integre le funzioni delle strutture cerebrali ad essa contigue. I principi fondamentali che il chirurgo deve tener presente sono principalmente due: la conoscenza dell'esatta posizione della lesione, dei rapporti che essa contrae con le strutture circostanti e la conoscenza delle funzioni cerebrali eventualmente coinvolte dalla patologia.

La neurochirurgia stereotassica permette di individuare esattamente piccole lesioni cerebrali sottocorticali o localizzate in aree cerebrali profonde attraverso la ricostruzione di coordinate tridimensionali. Il limite rappresentato dalla metodica stereotassica è dato dal volume del casco e dell'arco stereotassico che spesso ostacolano il chirurgo durante la fase di esposizione e di asportazione della lesione. Lo sviluppo dei nuovi sistemi di neuronavigazione ha permesso di ridurre significativamente la frequenza e l'entità dei deficit neurologici correlati al trattamento chirurgico, la durata dell'intervento, una minore esposizione della superficie cerebrale, il decorso in terapia intensiva e nei reparti di degenza. Sulla base di queste osservazioni il trattamento dei gliomi o delle recidive negli ultimi 20 anni è stato caratterizzato da indici di morbilità e mortalità sempre più bassi. Negli anni 70' la mortalità variava tra il 17 e il $27 \%$ e la morbidità tra il 35 e il $65 \%$ dei casi; negli ultimi anni questi valori si sono ridotti drasticamente e, infatti, attualmente la morbidità si aggira intorno al $8 \%$ mentre la mortalità, è di circa il $2.7 \%$ [5].

La neuronavigazione ha utilizzato le finalità della neurochirurgia stereotassica seguendo una linea di sviluppo autonoma; prescindendo dai calcoli fatti sugli assi coordinati cartesiani essa utilizza principi informatici derivanti dalle immagini TC e RM. I sistemi di neuronavigazione usano i dati digitali raccolti da TC e RM ad alta definizione 
che forniscono informazioni dettagliate di ciascun paziente utili durante il trattamento chirurgico. La neuronavigazione prevede una fase di preparazione del paziente, di trasferimento delle immagini acquisite sul sistema operativo, di pianificazione dell'intervento, di fusione dei dati e di neuronavigazione. Sulla superficie cutanea del paziente si dispongono markers cutanei adesivi in vicinanza dell'area d'interesse e a distanza in modo da avere un volume più ampio possibile e una riduzione della deformazione del volume endocranico. Dopo che il paziente ha eseguito la TC e/o la RM di base o con mezzo di contrasto, si provvede alla fase di trasferimento dei dati (via rete con immagini formato Dicom) sul computer che elaborerà un'immagine $2 \mathrm{D}$ e $3 \mathrm{D}$ (Fig. 1).

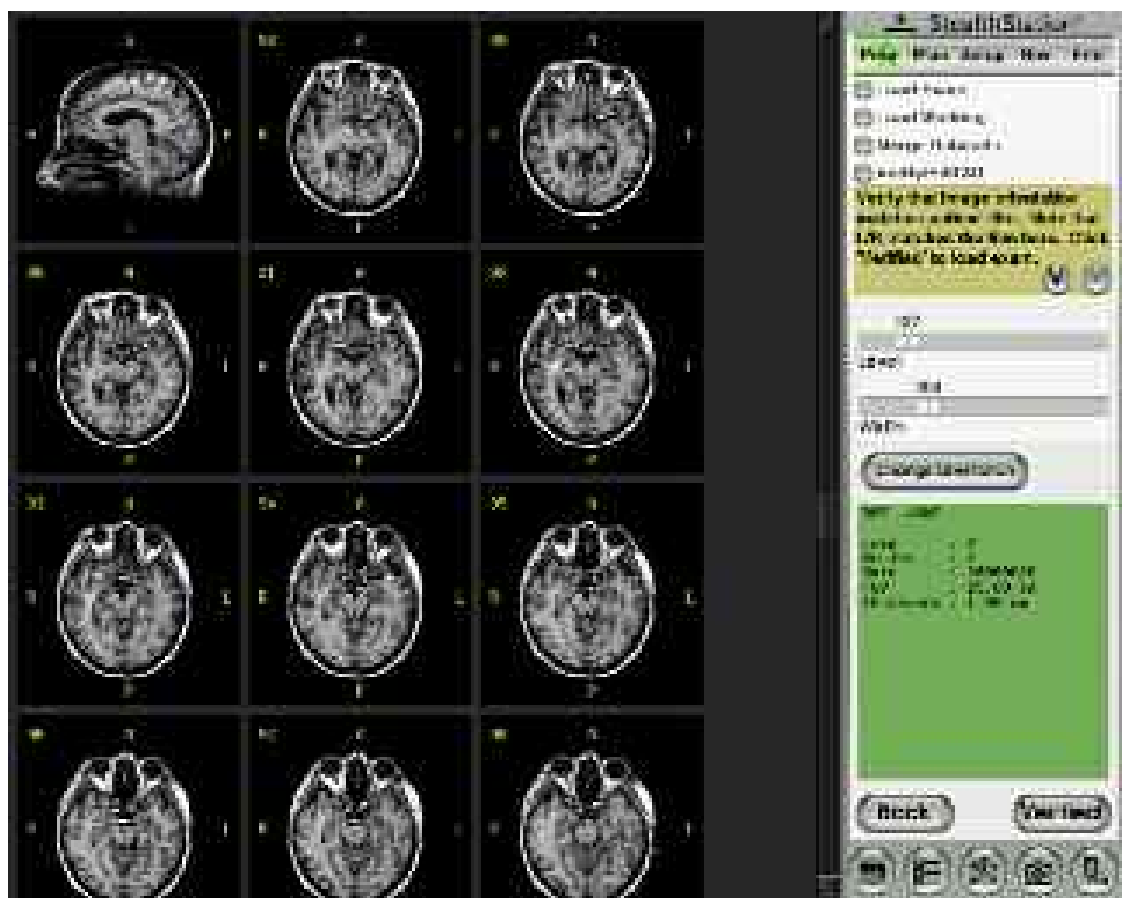

Fig 1.

Neuronavigazione: fase di trasferimento delle immagini $2 D$ e di ricostruzione $3 D$ in un sistema di neuronavigazione. 
I dati raccolti permettono la pianificazione dell'intervento alla consolle anche giorni prima del trattamento; per raggiungere una qualsiasi sede, anche se profonda o in area critica, è possibile programmare con il sistema di "surgical planning" il punto d'ingresso, il tragitto e il target rendendo possibili degli approcci anche più lunghi ma che passano attraverso aree non eloquenti e quindi poco rischiose per il paziente. Il neuronavigatore è dotato di una telecamera, di un sistema costituto da un arco interposto tra il paziente e la telecamera e da un pointer che, posizionato sui markers cutanei, permette di trasferire il volume d'interesse sul software e di ottenere la fusione dei dati e costruzione di immagini 3D. La fusione delle immagini può essere eseguita oltre che con l'ausilio dei markers cutanei (point merge), attraverso numerosi punti della superficie cranica (surface merge) o con la delimitazione punto per punto di uno specifico segmento fino a tracciarne il suo profilo (sculpture merge). L'errore di registrazione dato dalla telecamera è di $0.35 \mathrm{~mm}$ mentre quello di scansione è variabile tra 1 e $4 \mathrm{~mm}$ (Fig. 2).
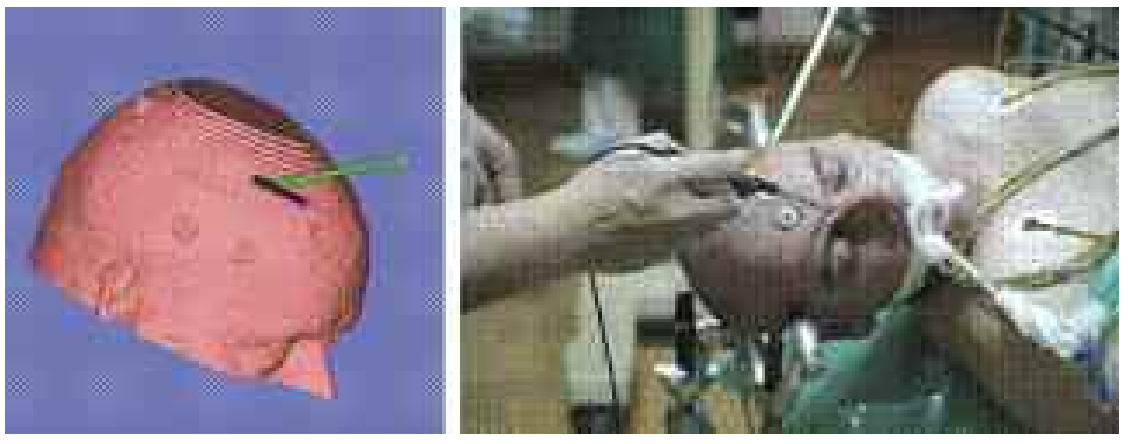

Fig 2.

Registrazione del paziente usando marcatori cutanei.

Nei sistemi di neuronavigazione è possibile l'integrazione di immagini utilizzate durante la fase diagnostica che documentano le caratteristiche metaboliche o le aree d'attivazione della zona corrispondente o periferica alla patologia d'interesse.

La Risonanza Magnetica Spettroscopica (MRS protonica), rappresenta una metodica che permette di analizzare i metaboliti cerebrali in relazione al loro contenuto di nuclei ${ }^{1} \mathrm{H}$. Il segnale ottenuto dai nuclei ${ }^{1} \mathrm{H}$ legati alla molecola dell'acqua è molto intenso e rende assai difficoltosa l'identificazione di segnali più deboli, provenienti dall'idrogeno 
legato ad altre molecole. Pertanto sono state impiegate con successo tecniche per la soppressione del segnale dell'acqua [6].

Recenti studi confermano il valore del N-Acetil Aspartato (NAA) come marker neuronale. Esso è esclusivamente documentato nei neuroni del cervello maturo. Nel cervello umano, NAA si riduce in situazioni caratterizzate da una riduzione quantitativa neuronale come ad esempio accade nelle patologie degenerative neuronali, nello stroke e nei tumori gliali. La possibilità di quantificare il depauperamento o l'alterazione neuronale in maniera specifica, rende la RMS un esame di valido supporto nello studio delle patologie cerebrali. Le alterazioni dei valori di altri metaboliti cellulari o della guaina mielinica come la colina (Cho) e la creatina $(\mathrm{Cr})$ sono ugualmente documentate nei tumori cerebrali [7].

La RM funzionale (RMf) consiste in un sistema di mappatura del parenchima cerebrale in grado di evidenziare, attraverso lo stato di ossidazione dell'emoglobina, i cambiamenti del flusso cerebrale e il consumo d'ossigeno derivante dall'attivazione neuronale [8]. Con questa tecnica è possibile studiare aree cerebrali coinvolte nel movimento, nella percezione sensitiva, nel linguaggio e in altre funzioni cognitive.

Con gli attuali sistemi di neuronavigazione è possibile fondere le immagini TC o RM morfologiche con la RMS protonica o la RMf. I vantaggi di tale fusione sono tali permettere durante la procedura chirurgica la continua visualizzazione di aree funzionalmente attive o di individuare porzioni di una neoplasia caratterizzate da una maggiore malignità. Quando il chirurgo deve trattare un caso in cui il tumore è localizzato in prossimità di aree eloquenti, come quella del linguaggio, la neuronavigazione con la RMf permette una significativa riduzione dei rischi operatori. Sono attualmente in corso studi riguardanti la reale correlazione tra una funzione cerebrale specifica e la corrispondente area di attivazione; le osservazioni finora eseguite dimostrano una buona affidabilità della RM funzionale. Il monitoraggio intraoperatorio delle funzioni corticali, in condizioni di awake anestesia, garantisce migliori risultati grazie alla possibilità di stimolare direttamente la corteccia cerebrale e delimitare l'area corticale funzionalmente attiva che in questo modo può essere completamente risparmiata [9].

Il microscopio operatorio (NC4, OME 8000, VM 900, OHS-1) può essere integrato nei sistemi di imaging intraoperatori e utilizzato quindi nella fase di localizzazione e trattamento di una lesione cerebrale. Esistono diversi sistemi operativi in cui in visione microscopica è possibile visualizzare i contorni della lesione o in cui il punto focale cor- 
risponde con la superficie della struttura da trattare (Sistemi HUD e PIP). Sono stati inoltre progettati sistemi di neuronavigazione in cui la telecamera è solidale con il microscopio riducendo eventuali ingombri sul campo operatorio.

La neuronavigazione è utilizzata, oltre che nel trattamento dei tumori cerebrali, anche nel campo della chirurgia vertebrale garantendo migliori risultati nel posizionamento dei mezzi di sintesi e riducendo l'esposizione del paziente e del personale di sala operatoria alle radiazioni ionizzanti.

I sistemi di fusione delle immagini sono oggi largamente utilizzati nel trattamento dei disturbi del movimento come il morbo di Parkinson, le distonie, differenti forme ipo o ipercinetiche e sindromi dolorose come la cefalea a grappolo (cluster headache). Il trattamento di questi disturbi prevede la cosiddetta Deep Brain Stimulation (DBS) che consiste nel posizionamento di un elettrodo sui nuclei cerebrali profondi funzionalmente o strutturalmente alterati. Nello studio di tali patologie è necessario un sistema di imaging che permetta la loro visualizzazione e localizzazione; la RM e la TC sono gli esami di prima scelta nella definizione di queste strutture. Le immagini ottenute secondo particolari accorgimenti (inclinazione del piano di scansione, spessore dei differenti piani, uso di marker esterni, scelta del piano di lavoro-assiale, frontale, sagittale-, eliminazione di artefatti), possono essere tra loro sovrapposte o fuse dal software. In questo modo è stato possibile sostituire, nella pratica chirurgica gli atlanti anatomici, che pur tenendo presente la variabilità topografica del nucleo d'interesse di ciascun paziente non ne permettono la loro visualizzazione diretta. Una volta che con il sistema di fusione delle immagini si localizza il nucleo da trattare è possibile determinare le sue coordinate sterotassiche che permetteranno il preciso posizionamento dell'elettrodo per la DBS [10] (Fig. 3).

La neuronavigazione rappresenta un sistema di localizzazione delle strutture anatomiche virtuali: le immagini utilizzate sono infatti quelle preoperatorie eseguite in una fase in cui il parenchima cerebrale è contenuto dalle strutture scheletriche. Questo sistema non considera pertanto le modificazioni spaziali del contenuto endocranico dopo l'esposizione della superficie cerebrale. Esistono delle situazioni in cui la perdita del liquido cefalorachidiano e la stessa asportazione della lesione cerebrale possono modificare la disposizione del volume cerebrale. Questo fenomeno, indicato con il termine "brain shift", non si osserva nel trattamento di piccole e medie lesioni (specialmente se superficiali) e nelle 


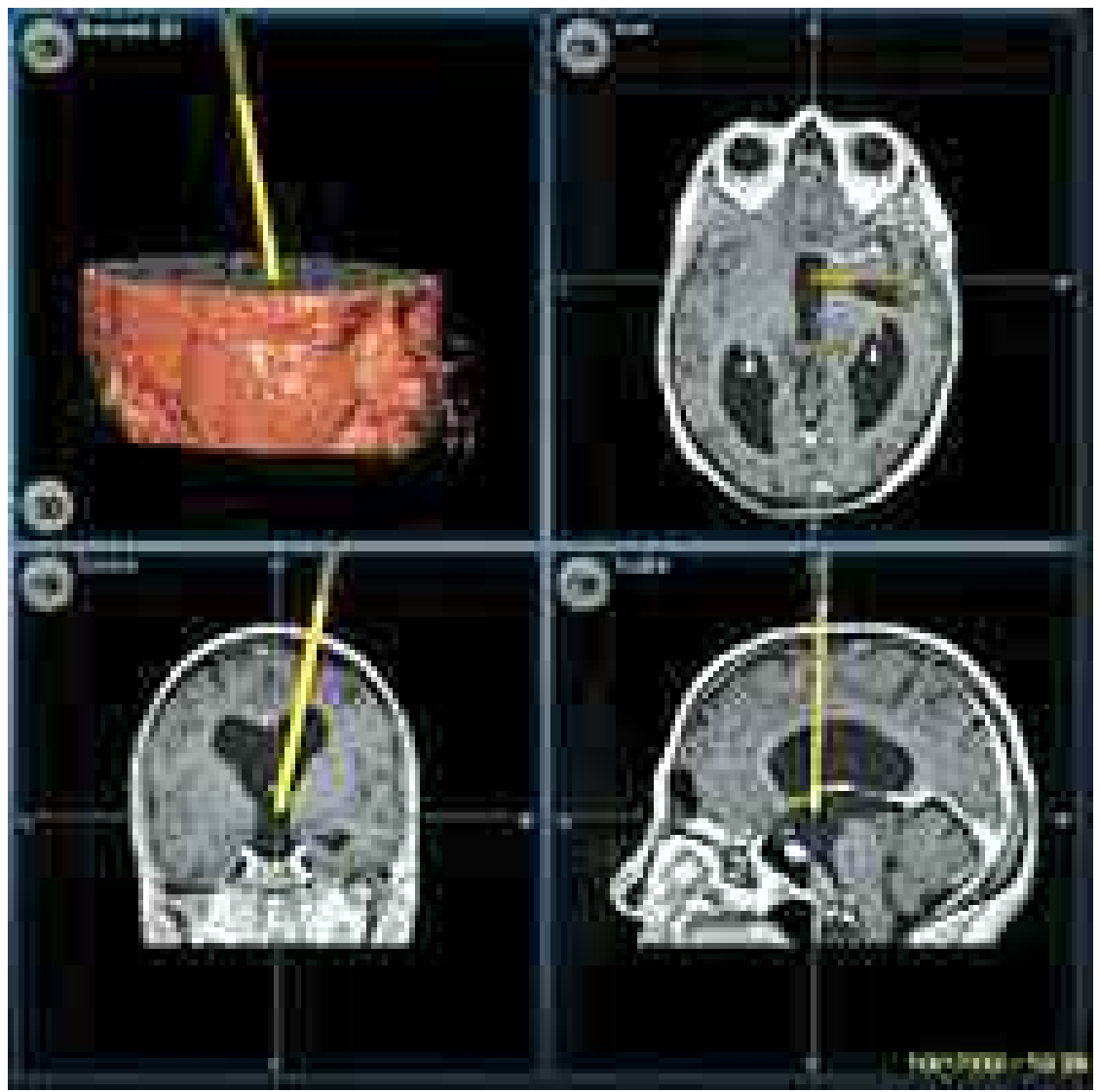

Fig 3 .

Localizzazione delle strutture anatomiche virtuali del paziente per la neuronavigazione.

patologie che sono in stretto rapporto con le strutture scheletriche o durali (ad esempio tumori del basicranio); è possibile però ridurre il brain shift limitando l'esposizione della superficie cerebrale, posizionando adeguatamente il paziente o riducendo la perdita di liquor. Per tale ragione, presso il Nostro Istituto, sono in corso degli studi che permettano di adattare il sistema di analisi dell'immagine alle modificazioni spaziali del parenchima cerebrale durante le differenti fasi d'intervento.

Negli ultimi anni è stato possibile introdurre nella pratica chirurgica l'uso della TC e della RM intraoperatoria che, a differenza dei sistemi di neuronavigazione virtuali, permette una visione del paren- 
chima cerebrale in tempo reale [11]. La RM intraoperatoria, generalmente di 0.5-tesla, permette un'analisi morfologica, metabolica e funzionale delle strutture da trattare. Il limite di tale sistema consiste negli alti costi della RM, di allestimento della sala operatoria con materiali esclusivamente amagnetici, nel maggiore impegno di risorse umane e nella limitazione dello spazio disponibile per il chirurgo.

La TC intraoperatoria (utilizzata presso il nostro Istituto) permette di acquisire immagini di buona qualità; le immagini raccolte possono essere confrontate e fuse con quelle di esami precedentemente eseguiti. Tale sistema permette la documentazione sul letto operatorio del risultato chirurgico e di eventuali complicanze postoperatorie. Il limite della TC consiste nella maggiore esposizione del personale di sala operatoria alle radiazioni ionizzanti.

L'ecografia intraoperatoria rappresenta un differente tipo di sistema che permette di ottenere immagini bidimensionali di qualità variabile in qualsiasi fase dell'intervento [12]. Con le attuali risorse tecnologiche è possibile integrare il sistema di raccolta delle informazioni $2 \mathrm{D}$ in tempo reale con il sistema di neuronavigazione $3 \mathrm{D}$ virtuale consentendo una più semplice localizzazione e asportazione della lesione.

Una metodica di chirurgia mini-invasiva è rappresentata dall'endoscopia con la quale è possibile raggiungere aree profonde con un minimo approccio chirurgico [13]. L'endoscopia trova la sua maggiore indicazione nel trattamento delle lesioni dei ventricoli cerebrali, di lesioni cistiche contigue ai ventricoli o di patologie della regione sellare.

La qualità dei sistemi di fibre ottiche permette di ottenere immagini bidimensionali ad alta definizione; l'affidabilità della tecnica endoscopica è aumentata dopo la sua introduzione nei sistemi di neuronavigazione. L'endoscopio può essere infatti trasformato in pointer e utilizzato nelle procedure di surgical planning. L'uso del navigatore facilita l'orientamento endoventricolare e il riconoscimento della lesione, non sempre facile in presenza di distorsione delle strutture anatomiche e quando questa sia ricoperta da ependima normale.

\section{ESPERIENZA CLINICA}

Dal 1978 ad oggi presso l'Istituto Neurologico Nazionale "Carlo Besta" (INNCB) sono stati sottoposti a trattamento chirurgico 4356 pazienti portatori di glioma sovratentoriale. Negli ultimi 5 anni 1500 pazienti 
sono stati trattati con l'ausilio di sistemi di neuronavigazione.

In corso di procedura chirurgica, il neuronavigatore è stato impiegato in 1090 interventi d'asportazione di glioma o glioma recidivo per via craniotomica, in 27 biopsie "frameless" a cielo coperto, in 18 biopsie per via endoscopica assistita, in 56 biopsie stereotassiche assistite, in 22 punture assistite di tumori cistici. I sistemi utilizzati sono stati: Radionics OTS (66 casi), Zeiss STN (23 casi), Sofamor Danek Stealth Station (328 casi), Philips Easy Guide (1270 casi). Il sistema di neuronavigazione è stato inoltre utilizzato in 40 procedure di neurochirurgia funzionale e in 8 fissazioni vertebrali assistite.

L'uso del sistema di neuronavigazione prevedeva, secondo le caratteristiche neuroradiologiche della lesione, l'esecuzione di una TC o RM con l'ausilio di markers cutanei. Questo tipo di procedura era realizzabile in corso di trattamento chirurgico per via craniotomica, stereotassica o endoscopica. L'entità dell'asportazione è stata sempre pianificata preoperatoriamente sulle immagini del neuronavigatore e verificate intraoperatoriamente.

I sistemi stereotassici utilizzati sono stati inizialmente il casco di Riechert e il BRW, più recentemente il casco di Leksell e il CRW.

La metodica neuroradiologica usata routinariamente per la scelta del target da biopsiare è stata la TC; nei casi in cui la TC non ha permesso una visualizzazione della lesione soddisfacente, si è preferito utilizzare la RM con casco stereotassico compatibile, o con metodiche computerizzate che permettessero la sovrapposizione e fusione delle immagini TC e RM. In alcuni gliomi di basso grado la scelta del target è stata effettuata attraverso l'ausilio della RM spettroscopica con lo scopo di evidenziare le aree della lesione a più alta cinetica cellulare caratterizzate da una più alto contenuto di colina.

La procedura di neuronavigazione è stata interrotta durante l'intervento per motivi tecnici in 6 casi $(0.54 \%)$. In tutti gli altri casi è stato possibile completare l'intervento rispettando il programma operatorio "virtuale" precedentemente stabilito sul neuronavigatore. L'asportazione è stata radiologicamente totale in 926 casi $(84.3 \%)$ e parziale in 172 casi $(15.6 \%)$. In 143 casi (13\%) l'intervento è stato eseguito per l'asportazione di lesioni residue o di recidive. I tumori operati erano stati giudicati per estensione o per sede inoperabili da altri centri neurochirurgici nel $7 \%$ dei casi. In nessun paziente si è evidenziato ai controlli postoperatori un residuo tumorale non previsto preoperatoriamente. L'accuratezza iniziale del sistema è stata mantenuta sempre inferiore ai 
$5 \mathrm{~mm}$. Il tempo richiesto per la messa a punto del sistema di navigazione in ogni intervento è stato di 15-30'. L'utilizzo del neuronavigatore ha consentito di utilizzare craniotomie più piccole (diametro sempre inferiore ai $4 \mathrm{~cm}$ ) ed approcci transulcali mirati alla lesione senza mai effettuare false traiettorie e permettendo l'identificazione del tumore anche nei casi in cui l'aspetto macroscopico era simile a quello del tessuto sano. Il cosiddetto "brain shift" ha introdotto un errore variabile a fine asportazione di 2-15 mm secondo la sede e dimensioni della lesione, l'entità della deliquorazione e della posizione del capo. Nel 15\% dei casi nel primo postoperatorio si sono manifestati deficit neurologici aggiunti. Tale percentuale è scesa al $7 \%$ al primo controllo ambulatoriale a 4-8 settimane dall'intervento; la mortalità a 30 giorni è stata del $1.4 \%$.

Negli ultimi 7 mesi sono stati eseguiti 24 interventi in awake anestesia; l'indicazione a questo tipo di anestesia dipendeva dalla contiguità della lesione ad aree eloquenti (linguaggio) e dalla necessità quindi di un monitoraggio intraoperatorio e di una delimitazione funzionale corticale. In tutti i pazienti è stata eseguita preoperatoriamente una RM funzionale la cui mappatura funzionale corrispondeva con quella ottenuta con la stimolazione corticale nel $70 \%$ dei casi. La completa conservazione del linguaggio è stata osservata nel $85 \%$ dei casi.

Le biopsie con assistenza del neuronavigatore sono state complessivamente 101 e così ripartite; frameless a cielo coperto 27 , con assistenza endoscopica 18 , stereotassica 56 . Il bersaglio prescelto è stato raggiunto in tutti i casi. La mortalità è stata nulla. Non si sono registrati deficit aggiuntivi a distanza. Gli errori di targeting nel gruppo 1 sono stati il 3\% e l'attendibilità diagnostica del $86 \%$.

\section{CONCLUSIONI}

I sistemi di neuronavigazione attualmente a disposizione permettono la fusione di immagini TC, RM, H-RMS, fRM pre operatorie con quelle TC e RM intraoperatorie. In questo modo è possibile ottenere immagini in tempo reale con il supporto di indagini preoperatorie più complesse. Lo sviluppo dei sistemi RM o TC potrà in un futuro prossimo permettere l'esecuzione di routine di uno studio funzionale o spettroscopico intraoperatorio on line.

La possibilità di utilizzare l'endoscopio, il microscopio operatorio robotizzato, o veri robot dotati di capacità specifiche "manuali”, per- 
metteranno uno standard di esecuzione di intervento eccellente e mininvasivo per molte patologie del Sistema Nervoso.

La tecnologia orientata verso il miglioramento di sistemi di supporto chirurgico non è però l'unica strada da percorrere; le scienze biomediche hanno infatti permesso lo sviluppo di metodiche che permettono una sempre più approfondita conoscenza dei meccanismi fisiopatologici e di soluzioni terapeutiche alternative alla chirurgia che comunque non può prescindere dalle qualità manuali e intellettive umane.

\section{BIBLIOGRAFIA}

[1] Spiegel EA, Wycis HT. Stereoencephalotomy. Part I. Methods and stereotaxis atlas of human brain. New York: Grune \& Stratton, 1952.

[2] Bennett AH, Godlee RJ. Excision of a tumor from the brain. Lancet 1884; 1090-1.

[3] Neuronavigation and Surgical Neurology: the beginning of a new age or the end of an old age? Surg. Neurol. 1999; 52: 7-12

[4] Dorward NL. Neuronavigation, the surgeon sextant. British Journal of Neurosurgery; 1997; 11(2): 101-103.

[5] Ciric I, Ammirati M, Vick N. Supratentorial gliomas: surgical consideration and immediate postoperative results. Gross total resection versus partial resection. Neurosurgery 1987; 22: 21-26.

[6] Gadian DG. Nuclear Magnetic Resonance and its applications to living systems, $2^{\text {nd }}$ edn. Oxford University Press, 1995.

[7] Preul MC, Camanos Z, Collins DL et al. Accurate, noninvasive diagnosis of human brain tumors by using proton magnetic resonance spectroscopy. Nature Medicine 1996; 2: 323-325.

[8] Lurito JT, Lowe M, Sartorius C, Mathews VP. Comparision of fMRI and intraoperative direct cortical stimulation in localization of receptive language areas. J Comput Assist Tomogr; 2000, 24: 99-105.

[9] Schulder M, Maldjaian JA, Liu WC et all. Functional MRI-guided surgery of intracranial tumors. Steractact Funct Neurosurg 1997; 68: 98-105.

[10] Lozano. Movement disorder surgery. Progress in Neurological Surgery. Ed Lunsford LD. (Karger) Vol 15.

[11] Alexander E, Moriarty T, Kikins R, Black P, Jolesz M. The present and 
the future of introperative MRI in neurosurgical procedures. Steractact Funct Neurosurg 1997; 68: 10-17.

[12] Giorgi C, Casolino DS. Preliminary clinical experience with intraoperative stereotactic ultrasound imaging. Steractact Funct Neurosurg 1997; 68: $54-58$.

[13] Broggi G, Dones I, Ferroli P, Franzini A, Servello D, Duca S. Image guided neuroendoscopy for third ventriculostomy. Acta Neurochir 2000; 142: 893-899. 